\title{
Percepção dos moradores do distrito de Eleutério, município de Itapira-SP, acerca da utilização de plantas medicinais
}

Perception of the residents of the district of Eleutério municipality of Itapira-SP on the use of medicinal plants Percepción de los habitantes del distrito de Eleuterio municipio de Itapira-SP acerca de la utilización de plantas medicinales Anderson MARTELLI ${ }^{1}$

Lucas Alan Hamaguti Bernardes de CARVALHO ${ }^{2}$

\author{
${ }^{I}$ Mestre; Programa de Pós graduação em Ciências Biomédicas - Centro Universitário Hermínio Ometto, FHO|Uniararas, Araras-SP; \\ Especialista em Laboratório Clínico pela Faculdade de Ciências Médicas-UNICAMP, Campinas-SP \\ ${ }^{2}$ Graduação em Tecnologia e Gestão Ambiental - Instituto Federal de Educação, Ciência e Tecnologia do Sul de Minas, Inconfidentes-MG
}

\section{Resumo}

A fitoterapia e o uso de plantas medicinais fazem parte da prática da medicina popular, que complementa o tratamento usualmente empregado para a população de menor renda. Essa utilização tem merecido crescente interesse, assim, a presente pesquisa investigou a percepção dos moradores de Eleutério, um Distrito Municipal de Itapira-SP, estritamente rural acerca da utilização de plantas medicinais. Participaram desta pesquisa 40 moradores de ambos os sexos, escolhidos de forma aleatória, com idade acima de 18 anos, residentes neste Distrito. Para tanto, a pesquisa foi realizada por meio de uma entrevista estruturada empregando um questionário, sendo os dados anotados em planilha para análise e comparação. Em relação ao uso de plantas medicinais, foram verificados que $80 \%$ da amostra fazem uso de alguma planta medicinal e $28 \%$ não seguem nenhum protocolo de preparo. Quanto aos possíveis efeitos tóxicos, $50 \%$ relatam que por serem natural não apresentam efeitos colaterais indesejáveis. Este estudo demonstra que a população deste distrito realiza a utilização de plantas medicinais com certa frequência, havendo a necessidade de uma maior divulgação quanto a essa prática, uma vez que, as pessoas em geral tem demonstrado interesse na fitoterapia como alternativa para o tratamento de diversas afecções.

Descritores: Fitoterapia; Plantas Medicinais; População Rural.

\section{Abstract}

Phytotherapy and the use of medicinal plants are part of the practice of folk medicine, which complements the treatment usually employed for the lower income population. This use has been of growing interest, so the present research investigated the perception of the residents of Eleutério, a Municipal District of Itapira-SP, strictly rural about the use of medicinal plants. A total of 40 randomly selected male and female residents of this age group aged 18 years and older participated in this study. For this, the research was performed through a structured interview using a questionnaire, the data being annotated in a spreadsheet for analysis and comparison. Regarding the use of medicinal plants, it was verified that $80 \%$ of the sample makes use of some medicinal plant and $28 \%$ do not follow any protocol of preparation. Regarding possible toxic effects, 50\% report that because they are natural they do not present undesirable side effects. This study demonstrates that the population of this district uses medicinal plants with some frequency, and there is a need for greater dissemination regarding this practice, since the general population has shown an interest in phytotherapy as an alternative for the treatment of several diseases.

Descriptors: Phytotherapy; Medicinal Plants; Rural Population.

\section{Resumen}

La fitoterapia y el uso de plantas medicinales forman parte de la práctica de la medicina popular, que complementa el tratamiento usualmente empleado para la población de menores ingresos. Esta utilización ha merecido creciente interés, así, la presente investigación investigó la percepción de los habitantes de Eleuterio, un Distrito Municipal de Itapira-SP, estrictamente rural acerca de la utilización de plantas medicinales. Participaron en esta investigación 40 residentes de ambos sexos, elegidos de forma aleatoria, con edad superior a 18 años, residentes en este Distrito. Para ello, la investigación fue realizada por medio de una entrevista estructurada empleando un cuestionario, siendo los datos anotados en planilla para análisis y comparación. En cuanto al uso de plantas medicinales, se verificó que el $80 \%$ de la muestra hace uso de alguna planta medicinal y el $28 \%$ no sigue ningún protocolo de preparación. En cuanto a posibles efectos tóxicos, el $50 \%$ informa que por ser natural no presentan efectos colaterales indeseables. Este estudio demuestra que la población de este distrito realiza la utilización de plantas medicinales con cierta frecuencia, habiendo la necesidad de una mayor divulgación en cuanto a esa práctica, una vez que la población en general ha demostrado interés en la fitoterapia como alternativa para el tratamiento de diversas condiciones.

Descriptores: Fitoterapia; Plantas Medicinales; Población Rural.

\section{INTRODUÇÃO}

Desde os primórdios da existência humana, os homens buscam na natureza recursos para melhorar suas próprias condições de vida, aumentando suas chances de sobrevivência. O emprego de plantas medicinais para a manutenção e a recuperação da saúde tem ocorrido ao longo dos tempos, mas à medida que as pessoas idosas vão morrendo, um arcabouço de conhecimento sobre as plantas medicinais estão sendo perdidas.

A utilização de plantas medicinais sempre esteve presente na sabedoria do senso comum, articulando a cultura de gerações, passadas e atuais, e a saúde, uma vez que estes aspectos não ocorrem isoladamente, mas estão inseridos em um contexto histórico $^{1,2}$.

Ao longo dos séculos, substâncias de origem vegetal constituíram as bases para o tratamento de um número variado de doenças, quer de forma tradicional pelo conhecimento das propriedades de determinada planta, que é passado de geração a geração, quer pela utilização de espécies vegetais, como fonte de moléculas ativas ${ }^{3}$.

Atualmente o uso de plantas medicinais e fitoterápicos ocorrem por todo o mundo e, no Brasil, Mello et al. $^{4}$ retratam que esta prática vem da miscigenação cultural envolvendo africanos, europeus e indígenas. Por isso, essa cultura é tão diversa, e para muitas dessas comunidades brasileiras, é o único recurso terapêutico disponível. Dentre as principais razões que impulsionam o grande crescimento do mercado mundial de fitoterápicos a cada ano destaca-se a valorização de hábitos de vida mais saudáveis e, consequentemente, o consumo de produtos naturais; os evidentes efeitos colaterais dos medicamentos sintéticos; a descoberta de novos princípios ativos de plantas e a 
comprovação científica de suas atividades terapêuticas 5 .

Devido ao fato do Brasil ser detentor de vasta biodiversidade tanto faunística como florística, além de grande variedade étnica e cultural, a população brasileira, principalmente a residente em zonas rurais, utiliza comumente o seu conhecimento empírico acerca do potencial terapêutico de plantas medicinais $^{6,7}$, fator que estimulou a realização desta pesquisa junto aos moradores de Eleutério, um distrito rural do município de Itapira-SP.

O uso de plantas medicinais é mencionado desde a pré-história, quando eram utilizadas na forma de cataplasmas, com o intuito de estagnar hemorragias e favorecer o reparo tecidual, sendo muitas dessas plantas ingeridas, para atuação em via sistêmica $^{8}$. Inúmeras plantas com poder medicinal são empregadas no tratamento de diferentes enfermidades ${ }^{9}$, dentre elas, a cicatrização de feridas. Paradoxalmente, é possível encontrar no Brasil um grande número de pessoas que não possuem acesso ao tratamento convencional e muitas vezes essas lesões podem promover alterações funcionais importantes.

Apesar dos avanços da medicina moderna no mundo, a Organização Mundial da Saúde reconhece que a maioria da população de países em desenvolvimento depende da medicina tradicional, já que $80 \%$ desta população desenvolvem práticas tradicionais em seus cuidados básicos na saúde, sendo $85 \%$ dessas representadas pelo uso de plantas medicinais ${ }^{10}$.

É possível observar no contexto brasileiro que as pesquisas com plantas medicinais apresentaram avanços significativos nas últimas décadas. Dutra et al. ${ }^{11}$ retrataram que nos últimos 28 anos (entre os anos de 1987 e 2015), cientistas brasileiros publicaram 34.614 artigos sobre as plantas medicinais. Entre 2011 e 2013 foram publicados mais de 10.000 artigos científicos sobre o tema.

Diante desses dados, acreditamos que este estudo torna-se relevante e oportuno à medida que a incidência e prevalência de inúmeras patologias vêm aumentando, com consequências sociais significativas sobre os portadores que podem desenvolver sequelas crônicas, sendo a utilização de plantas medicinais uma alternativa de tratamento pouco dispendioso comparado aos fármacos sintéticos e mais acessíveis à população carente.

Assim, o objetivo deste estudo foi investigar o conhecimento, formas de utilização das plantas medicinais e os motivos desse uso junto aos moradores de Eleutério, um Distrito Municipal, estritamente rural, afastado $20 \mathrm{~km}$ da área urbana de Itapira-SP e que não apresenta nenhum estabelecimento farmacêutico favorecendo a disseminação desse conhecimento e o direcionamento de novas pesquisas.

\section{MATERIAL E MÉTODO}

Trata-se de uma investigação de caráter quantitativo, transversal, observacional e descritivo, realizado seguindo as normas que regulamentam a pesquisa em seres humanos, contidas na Resolução $\mathrm{n}^{\circ}$ 466/12 do Conselho Nacional de Saúde, aprovado pelo Comitê de Ética e Pesquisa da Faculdade Municipal Professor Franco Montoro, tendo recebido parecer favorável sob o registro CEP/FMPFM: 2.720.358. A amostra foi composta por 40 moradores de ambos os sexos, escolhidos de forma aleatória, com idade acima de 18 anos, residentes no Distrito municipal de Eleutério, município de Itapira-SP.

A pesquisa foi realizada por meio de uma entrevista estruturada, ocorrido nas residências de cada pessoa selecionada com o emprego de um questionário abordando dados pessoais, utilização de plantas medicinais e seus motivos, formas de aplicação dentre outros fatores. Após os esclarecimentos sobre os objetivos e a metodologia da pesquisa, os que concordaram em participar assinaram o Termo de Consentimento Livre e Esclarecido (TCLE) conforme preconizado na legislação vigente.

Como critério de inclusão da amostra na pesquisa foram analisadas informações comprobatórias como: residir a mais de 10 anos neste vilarejo, idade acima de 18 anos e o aceite formal em participar da pesquisa com a assinatura do TCLE. Como critério de exclusão, não residir a mais de 10 anos neste local, idade inferior a 18 anos e pelo não consentimento em participar da pesquisa.

Após a coleta de dados, as informações foram digitadas e tabuladas em banco de dados do programa Microsoft Excel - 2010 para as análises estatísticas de frequência, média e desvio padrão. Posteriormente foi realizada uma comparação dos resultados observados com os descritos na literatura.

\section{RESULTADOS E DISCUSSÃO}

Foram pesquisados 40 moradores do distrito de Eleutério, município de Itapira-SP. A amostra foi composta por 26 homens e 14 mulheres, com faixa etária entre 18 a 62 anos de idade, médio e desvio

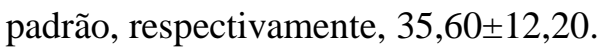

A utilização de plantas medicinais faz parte da prática da medicina popular, que pode ou não complementar o tratamento usualmente empregado para a população de menor renda. Em relação ao uso de plantas medicinais na amostra pesquisada, foi verificado que $80 \%(n=32)$ fazem uso de alguma planta medicinal (Figura 1A). Os dados corroboram com estudo etnobotânico e etnofarmacológico realizado por Ferrão ${ }^{12}$ no município de Buritis, região Noroeste de Minas Gerais, com 393 indivíduos entrevistados, sendo que $91 \% \quad(n=359)$ afirmaram fazer uso de plantas para tratamento de 
alguma enfermidade e/ou possuem conhecimentos acerca das mesmas.

Apesar da importância da utilização da fitoterapia no combate a inúmeros quadros patológicos, o fato de sua utilização ser geralmente de forma empírica e as preparações na maioria das vezes, não seguirem padrões sistemáticos de controle de qualidade, alguns protocolos terapêuticos envolvendo essa prática podem resultar em efeitos adversos ao paciente ${ }^{13}$. Neste sentido, aos entrevistados que relataram fazer uso de alguma planta medicinal, foi questionado sobre algum tipo de protocolo de preparo das infusões, sendo constatado que $28 \%(\mathrm{n}=9)$ seguem algum protocolo padronizado de preparo; $72 \%(\mathrm{n}=23)$ relataram seguir receita própria (Figura 1B). Em referência a via de administração do preparado, $97 \%(\mathrm{n}=31)$ relataram ser por via oral e $3 \%(n=1)$ relataram fazer uso por outras vias não sendo informado pelo entrevistado.

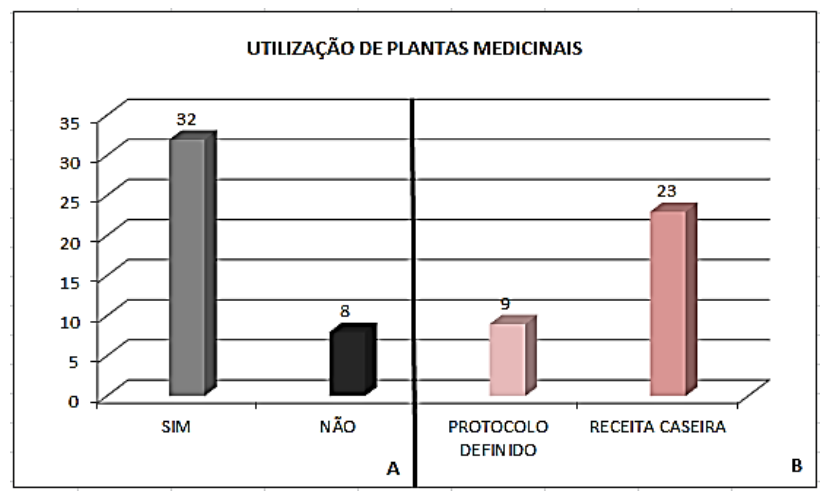

Figura 1. A. Utilização de plantas medicinais pela amostra. B. Protocolo de preparo utilizando essas plantas.

Complementando as informações, a amostra foi indagada sobre os perigos das interações medicamentosas, efeitos colaterais e tóxicos quanto à utilização das plantas medicinais, sendo observado que $50 \%(n=20)$ relataram que por serem natural não fazem mal e não apresentam efeitos colaterais indesejáveis; $42 \%(\mathrm{n}=17)$ fazem menos mal que os medicamentos convencionais/alopáticos, mas que devem ter cautela em seu uso e $8 \%(n=3)$ faz tão mal quanto um medicamento alopático (Figura 2). Na coleta desse dado, foi perceptível no entendimento da amostra que, os medicamentos industrializados causam mais efeitos adversos reforçando que as plantas medicinais são melhores por não apresentarem tais efeitos. Arnous et al. ${ }^{14}$ entrevistando 500 famílias sobre a utilização de plantas medicinais verificaram que $61,2 \%$ dessa amostra afirmaram que este tipo de tratamento não faz mal à saúde.

Porém, o uso imprudente, muitas vezes até para finalidades diferentes daquelas tradicionalmente empregadas, sua identificação errônea, a presença de adulterantes e contaminantes, as associações e sinergismos possíveis, assim como, a obtenção ou preparo indevido podem causar sérios problemas de saúde pública sendo importante evitar a automedicação ou o uso indiscriminado, uma vez que o emprego de plantas medicinais ou mesmo de medicamentos fitoterápicos não significa ausência de efeitos colaterais e de reações adversas ou tóxicas ${ }^{15}$. Veiga Júnior et al. ${ }^{16}$ revelam que entre os danos ocasionados por algumas plantas estão a hepatotoxicidade, nefrotoxicidade, genotoxicidade, incidência aumentada de tumores, efeitos abortivos, lesão ao sistema nervoso central, entre outros.

Oliveira e Dalla ${ }^{17}$ apontam que, dentre as publicações relacionadas às plantas nativas brasileiras e de amplo uso popular, são retratados riscos da associação com medicamentos, levando a alterações farmacológicas e toxicológicas como, por exemplo, alteração das taxas de glicemia e da pressão arterial.

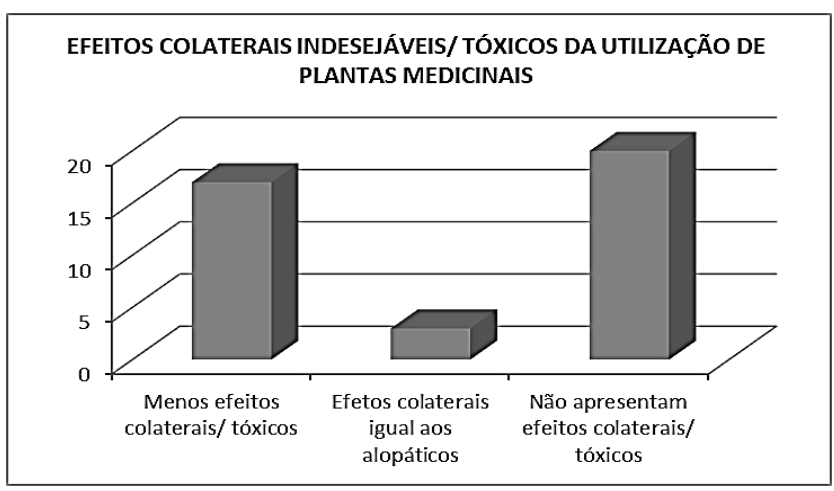

Figura 2. Interações medicamentosas, efeitos colaterais e tóxicos quanto à utilização das plantas medicinais.

Quando questionadas sobre quais plantas utilizam, 15 plantas foram mencionadas (Figura 3). Das plantas citadas, o boldo - Plectranthus barbatus foi mencionado 15 vezes (27\%), seguido pela hortelã - Mentha spicata (18\%) e a erva cidreira - Melissa officinalis (11\%) (Figura 3). Esses dados foram observados em um estudo realizado por Maravai et $a l .{ }^{18}$ com usuários de quatro unidades de Estratégia de Saúde da Família do município de Criciúma/SC, totalizando 672 famílias onde o boldo foi mencionado 173 vezes $(16,75 \%)$, seguido pela hortelã $(10,26 \%)$.

Diante desses dados, Zeni et al. ${ }^{19}$ retratam que a utilização de plantas medicinais não devem ser consideradas livres de riscos, pois entre as plantas citadas como o maracujá, da espécie Passiflora officinalis, pode interagir com hipnóticos e ansiolíticos; o alho Allium sativum diminui os níveis plasmáticos de medicamentos usados para tratar pacientes com HIV e pode causar dermatite alérgica, náuseas e vômitos; a hortelã, da espécie Mentha spicata, é responsável por causar dermatite alérgica; o boldo, da espécie Plectranthus barbatus, pode ser nefrotóxico, enquanto o gengibre Zingiber officinale possui propriedades abortivas.

Complementando as informações sobre a utilização das plantas medicinais, a amostra foi questionada sobre quais os motivos que as levaram à 
utilização dessas plantas, sendo citado na Tabela 1. Fazendo referência a esses motivos, foi realizada uma comparação da indicação terapêutica descrita na literatura, observando-se que vários motivos alegados pela amostra são condizentes com o descrito na literatura podendo ser visualizado na última coluna desta tabela.

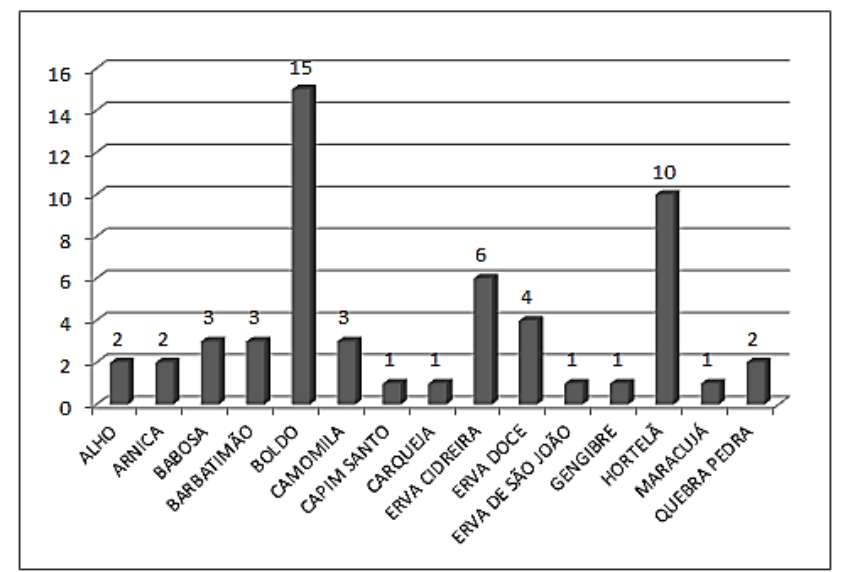

Figura 3: Plantas medicinais utilizadas pelos moradores do distrito de Eleutério, municipio de Itapira-SP.

Tabela 1. Distribuição das plantas utilizadas, motivo do uso pela amostra e a indicação terapêutica descrito na literatura

\begin{tabular}{|c|c|c|c|}
\hline $\begin{array}{l}\text { PLANTA } \\
\text { UTILIZADA }\end{array}$ & $\begin{array}{c}\text { NOME } \\
\text { CIENTÍFICO }\end{array}$ & $\begin{array}{c}\text { USO DA } \\
\text { PLANTA NA } \\
\text { AMOSTRA } \\
\text { PESQUISADA } \\
\end{array}$ & $\begin{array}{l}\text { INDICAÇÃO } \\
\text { TERAPÊUTICA }\end{array}$ \\
\hline ALHO & Allium sativum & Anti bacteriano & $\begin{array}{l}\text { Coadjuvante no } \\
\text { tratamento de } \\
\text { hiperlipidemia, } \\
\text { hipertensão arterial leve, } \\
\text { sintomas de gripes e } \\
\text { resfriados e prevenção da } \\
\text { aterosclerose* }\end{array}$ \\
\hline ARNICA & $\begin{array}{l}\text { Arnica } \\
\text { montana }\end{array}$ & Dor muscular & $\begin{array}{l}\text { Anti-inflamatório em } \\
\text { contusões e distensões, } \\
\text { equimoses e hematomas* }\end{array}$ \\
\hline BABOSA & Aloe vera & $\begin{array}{l}\text { Estômago, } \\
\text { queimadura }\end{array}$ & Cicatrizante $^{*}$ \\
\hline BARBATIMÃO & $\begin{array}{l}\text { Stryphnodendr } \\
\text { on adstringens }\end{array}$ & Cicatrização & Cicatrizante* \\
\hline BOLDO & $\begin{array}{l}\text { Plectranthus } \\
\text { barbatus }\end{array}$ & $\begin{array}{l}\text { Estômago, } \\
\text { ressaca alcoólica, } \\
\text { má digestão, } \\
\text { enjoo }\end{array}$ & Antidispéptico ${ }^{*}$ \\
\hline CAMOMILA & $\begin{array}{l}\text { Chamomilla } \\
\text { recutita }\end{array}$ & $\begin{array}{l}\text { Cólicas } \\
\text { abdominais, } \\
\text { relaxante }\end{array}$ & $\begin{array}{l}\text { Antiespasmódico, } \\
\text { ansiolítico e sedativo } \\
\text { leve. Uso externo: anti- } \\
\text { inflamatório* }\end{array}$ \\
\hline CAPIM SANTO & & Relaxante & $\begin{array}{l}\text { Antiespasmódico, } \\
\text { ansiolítico e sedativo } \\
\text { leve }\end{array}$ \\
\hline CARQUEJA & $\begin{array}{l}\text { Baccharis } \\
\text { trimera }\end{array}$ & Diabetes & Antidispéptico* \\
\hline $\begin{array}{l}\text { ERVA } \\
\text { CIDREIRA }\end{array}$ & $\begin{array}{l}\text { Melissa } \\
\text { officinalis }\end{array}$ & $\begin{array}{l}\text { Dor abdominal, } \\
\text { constipação } \\
\text { intestinal }\end{array}$ & $\begin{array}{l}\text { Antiespasmódico, } \\
\text { ansiolítico e sedativo } \\
\text { leve }\end{array}$ \\
\hline ERVA DOCE & $\begin{array}{l}\text { Pimpinella } \\
\text { anisum }\end{array}$ & $\begin{array}{l}\text { Relaxante, } \\
\text { melhora } \\
\text { digestão }\end{array}$ & $\begin{array}{ll}\text { Antidispéptico } & \text { e } \\
\text { antiespasmódico* }\end{array}$ \\
\hline $\begin{array}{l}\text { ERVA DE SÃO } \\
\text { JOÃO }\end{array}$ & $\begin{array}{l}\text { Hypericum } \\
\text { perforatum }\end{array}$ & Não informado & $\begin{array}{l}\text { antiinflamatório, } \\
\text { sedativo, analgésico, } \\
\text { diurético, queimaduras, } \\
\text { reumatismo, } \\
\text { hemorroidas, diarreia, } \\
\text { histeria e depressão }{ }^{* *}\end{array}$ \\
\hline GENGIBRE & $\begin{array}{l}\text { Zingiber } \\
\text { officinale }\end{array}$ & $\begin{array}{l}\text { Infecção } \\
\text { garganta }\end{array}$ & $\begin{array}{l}\text { Antiemético, } \\
\text { antidispéptico, } \\
\text { expectorante e nos casos } \\
\text { de cinetose }\end{array}$ \\
\hline HORTELÃ & Mentha spicata & $\begin{array}{ll}\text { Relaxante, } & \text { dor } \\
\text { abdominal, } & \\
\text { melhora } & \text { da } \\
\text { digestão, } & \\
\text { cefaleia, } & \\
\text { constipação } & \\
\text { intestinal } & \\
\end{array}$ & $\begin{array}{l}\text { Má digestão, enjoo } \\
\text { ou vômitos, calmantes e } \\
\text { expectorantes* }\end{array}$ \\
\hline MARACUJÁ & $\begin{array}{l}\text { Passiflora } \\
\text { incarnata }\end{array}$ & Relaxante & $\begin{array}{lll}\begin{array}{l}\text { Ansiolítico } \\
\text { leve* }^{*}\end{array} & \text { sedativo } \\
\end{array}$ \\
\hline $\begin{array}{l}\text { QUEBRA } \\
\text { PEDRA }\end{array}$ & $\begin{array}{l}\text { Phyllanthus } \\
\text { niruri }\end{array}$ & $\begin{array}{l}\text { Cálculos renais, } \\
\text { cólicas renais }\end{array}$ & $\begin{array}{l}\text { Litolítico nos casos de } \\
\text { litíase urinária* }\end{array}$ \\
\hline
\end{tabular}

Quanto à prescrição e/ou indicação do uso de alguma planta medicinal na amostra que realizou ou realiza o uso desta, $62 \%(\mathrm{n}=20)$ detém o conhecimento que foi passado por familiares e passou a utiliza-la; 26\% $(\mathrm{n}=8)$ recebeu indicação de amigos/vizinhos e $6 \%(\mathrm{n}=2)$ de profissional de saúde (médico, enfermeiro ou farmacêutico)( $\mathrm{n}=2)$.Por fim, $6 \%(n=2)$ ficou conhecendo através dos meios de comunicação (televisão, jornais, revistas, internet). Estudo realizado por Alves e Silva ${ }^{22}$ demonstrou que o conhecimento das plantas medicinais por parte de uma comunidade vinha $11 \%$ por meio de vizinhos, $8 \%$ com profissionais de saúde, $5 \%$ de acordo com crenças ou tradições, $57 \%$ com pais ou avós, $1 \%$ em livros ou revistas e $18 \%$ em outras fontes. Arnous et al. $^{14}$ verificaram que $84,5 \%$ de sua amostra afirmaram ter aprendido sobre as plantas medicinais com seus ascendentes (pais e avós principalmente) e apenas uma pessoa $(0,2 \%)$ relatou ter aprendido com um profissional de saúde, corroborando com os dados observados nesta pesquisa.

O percentual de conhecimento em referência às plantas medicinais diminui gradativamente com a redução da faixa etária dos indivíduos. Neste sentido, a amostra foi questionada sobre qual a faixa etária detém os maiores conhecimentos sobre a utilização dessas plantas, sendo observado que $58 \%(\mathrm{n}=23)$ afirmaram que os idosos apresentam maiores conhecimentos sobre este tema, seguido dos adultos $28 \%(n=11)$, jovens $12 \%(n=5)$ e crianças $2 \%(n=1)$. Segundo Costa et al. ${ }^{23}$, à medida que idosos vão desaparecendo, verdadeiros arquivos deixam de existir. Lima et al. ${ }^{24}$ e Feijó et al. ${ }^{25}$ relatam que o conhecimento sobre a utilização terapêutica das plantas medicinais é, normalmente, uma característica presente na população mais idosa, sendo que estas plantas são utilizadas principalmente para o tratamento de morbidades menores. Ferrão ${ }^{12}$ observou que a parte da população entrevistada em seu estudo que mais detêm o conhecimento sobre as plantas medicinais encontrava-se acima dos 60 anos: $100 \%$ dos indivíduos nessa faixa afirmaram possuir conhecimento acerca de plantas medicinais.

Este fato retrata menor atenção da população mais jovem quanto ao conhecimento transmitido através das gerações, mesmo que pessoas desta faixa etária tenham acesso à escolaridade ${ }^{26}$. Medeiros et al. $^{27}$ enfatizam que os meios modernos de comunicação tem favorecido a perda desse conhecimento sobre o uso de plantas, reforçando a importância de trabalhos que resgatem o conhecimento etnofarmacológico da população mais velha junto a população mais jovem.

Em referencia ao cultivo de algum tipo de planta medicinal, 33\% $(n=13)$ relataram fazer o cultivo de alguma planta com potencial terapêutico. Esperávamos um numero maior de pessoas que realizassem o cultivo de alguma planta medicinal, 
uma vez se tratando de um distrito estritamente rural. Dentre as plantas cultivadas nos quintais das residências citadas nesta pesquisa aparecem o boldo (Plectranthus barbatus), hortelã (Mentha spicata), erva doce (Pimpinella anisum) e a babosa (Aloe vera).

Um estudo realizado por Lima et al. ${ }^{24}$ verificou que os homens idosos possuem o hábito de cultivar as plantas medicinais em seus quintais e jardins, como também as adquirem de vizinhos, amigos, e até em lugares alagadiços/pantanosos (popularmente conhecidos como o "brejo") perto de onde moram, podendo trazer riscos relacionados à qualidade e à segurança, já que no ato de obtenção pode ocorrer a identificação errônea da planta. Os mesmos autores reforçam que uma das estratégias para atenuar esse risco seria a adoção de hortos de plantas medicinais, cujas espécies seriam certificadas por botânicos, e sua indicação e uso ficariam sob a responsabilidade de equipe de saúde multiprofissional. Neste sentido, Lorenzetti et al. ${ }^{28}$ retratam que o cultivo de plantas medicinais é uma prática muito comum em cidades do interior, nas quais são desenvolvidas hortas medicinais em escolas, bairros e no próprio quintal garantindo uma pequena produção para atender a demanda de uma pequena parcela da população familiar ou local sem fins lucrativos.

\section{CONCLUSÃO}

A partir dos resultados obtidos, foi verificado que a utilização de plantas medicinais nesta amostra está incorporada e bem difundida representando uma forma de recurso terapêutico complementar diante de algumas afecções. A transmissão dos conhecimentos em relação à fitoterapia vem ocorrendo principalmente através de seus ascendentes e os idosos são as pessoas que detém o maior conhecimento. Neste sentido, as linhas de pesquisas nesta área devem evoluir quanto à utilização e validação do potencial terapêutico das plantas medicinais para que possam ser retransmitidas de forma segura às futuras gerações.

As representações sobre as plantas medicinais estão aportadas na compreensão de segurança, visto que, por serem naturais, teriam menor probabilidade de desencadear reações tóxicas ficando evidenciado o falta de conhecimento sobre as interações medicamentosas podendo interferir na eficácia dos medicamentos utilizados nos tratamentos de outras patologias. Em suma, este estudo contribuiu em aumentar o conhecimento sobre a fitoterapia junto ao conhecimento popular e os cuidados de sua utilização, sendo necessário que as plantas em uso devam ser certificadas por profissionais habilitados evitando possíveis erros e sua prescrição realizada por uma equipe de saúde multiprofissional qualificada.

\section{REFERÊNCIAS}

1. Maciel MA, Pinto AC, Veiga Jr VF, Grinberg NF, Echevarria A. Plantas medicinais: a necessidade de estudos multidisciplinares. Quim Nova. 2002;15(3):429-38.

2. Alvim NAT, Ferreira MA, Cabral IE, Almeida Filho AJ. . et al. O uso de plantas medicinais como recurso terapêutico: das influências da formação profissional às implicações éticas e legais de sua aplicabilidade como extensão da prática de cuidar realizada pela enfermeira. Rev Latino-Am Enfermagem. 2006;14(3):316-23.

3. Carvalho ACB, Silveira D. Drogas vegetais: uma antiga nova forma de utilização de plantas medicinais. Brasília Med. 2010;48(2):219-37.

4. Melo JG, Martins JDGR, Amorim ELC, Albuquerque UP. Qualidade de produtos a base de plantas medicinais comercializados no Brasil: castanha-da-índia (Aesculus hippocastanum (L.), capim-limão (Cymbopogon citratus (DC.) Stapf e centela (Centella asiatica (L.) Urban). Acta Bot Bras. 2007;21(1):27-36.

5. Secretaria Municipal de Saúde de Londrina: Protocolo De Fitoterapia. 3. Ed; 2012.

6. Silva LAF, Silva JA, Lima CRO, Dambros CE, Cardoso VS. Uso popular do barbatimão. In: Silva LAF, Eurides D, Paula JR, Lima CRO, Moura MI. Manual do barbatimão. Goiânia: Kelps;2010.

7. Corrêa VS, Cerdeira AL, Fachin AL, Bertoni BW, Pereira PS, França SC et al. Geographical variation and quality assessment of Stryphnodendron adstringens (Mart.) Coville within Brazil. Genet Resour Crop Evol. 2012;59(7):1349-56.

8. Silva DM, Mocelin KR. O cuidado de enfermagem ao cliente portador de feridas sob a ótica do cuidado transcultural. Nursing. 2007;9(105):8188.

9. Rodrigues DF, et al. O extrato da casca de barbatimão, Stryphnodendron adstringens (Martius) Coville, na cicatrização de feridas em animais. Enciclopédia Biosfera, Centro Científico Conhecer - Goiânia. 2013;9(16):1583-1601.

10.Carvalho ACB, Nunes DSG, Baratelli TG, Shuqair NSMSAQ, Machado Netto E. Aspectos da legislação no controle dos medicamentos fitoterápicos. T \& C Amazônia. 2007;5(11):26-32.

11.Dutra RC, Campos MM, Santos AR, Calixto JB. Medicinal plants in Brazil: Pharmacological studies, drug discovery, challenges and perspectives. Pharmacol Res. 2016;112:4-29.

12.Ferrão BH, Oliveira HB, Molinari RF Teixeira MB, Fontes GG, Amaro MOF et al. Importância do conhecimento tradicional no uso de plantas medicinais em Buritis, MG. Brasil. Ciência e Natura. 2014;36(Ed Especial):321-34.

13.Turolla MSR, Nascimento ES. Informações 
toxicológicas de alguns fitoterápicos utilizados no Brasil. Rev Bras Ciênc Farm. 2006;42(2):289-306.

14. Arnous AH, Santos AS, Beinner RPC. Plantas medicinais de uso caseiro - conhecimento popular e interesse por cultivo comunitário. Espaç Saúde. 2005;6(2):1-6.

15.Araújo EJF, Araújo DYML, Freitas R, Ferreira PMP. Aspectos toxicológicos da planta medicinal Casearia sylvestris Swartz: revisão de literatura. Rev Ciênc Farm Básica Apl. 2014;35(3):355-61.

16. Veiga Júnior FV, Pinto AC, Maciel MAM. Plantas medicinais: cura segura? Quim Nova. 2005;28(3):519-28.

17. Oliveira AE, Dalla Costa $\mathrm{T}$. Interações farmacocinéticas entre as plantas medicinais Hypericum perforatum, Gingko biloba e Panax gingseng e fármacos tradicionais. Acta Farm Bonaerense. 2004;23(4):567-78.

18. Maravai SG, Costa CS, Lefchako FJ, Martinello OB, Becker IRT, Rossato AE. Plantas medicinais: percepção, utilização e indicações terapêuticas de usuários da estratégia saúde da família do município de Criciúma- SC vinculados ao PETSaúde. Arq Catarin Med. 2011;40(4):69-75.

19.Zeni ALB, Parisotto AV, Matos GM, Helena ETS. Utilização de plantas medicinais como remédio caseiro na Atenção Primária em Blumenau, Santa Catarina, Brasil Ciênc saúde coletiva. 2017;22(8):2703-12.

20.Brasil. Agência Nacional de Vigilância Sanitária. Formulário de Fitoterápicos da Farmacopéia Brasileira / Agência Nacional de Vigilância Sanitária. Brasília: Anvisa, 2011.

21. Chiovatto RD, Fukuda EY, Feder D, Nassis CZ. Fluoxetina ou Hypericum perforatum no tratamento de pacientes portadores de transtorno depressivo maior leve a moderado? Uma revisão. Arq Bras Ci Saúde. 2011;36(3):168-75.

22. Alves DL, Silva CR. Fitohormônios: abordagem natural da terapia hormonal. São Paulo: Atheneu; 2002.

23. Costa FGC, Nunes FCP, Peres V. Mapeamento etnofarmacológico e etnobotânico de espécies de cerrado, na microrregião de Patos de Minas. Perquirere. Patos de Minas: UNIPAM. 2010;7(2):93-111.

24.Lima SCS, Arruda GO, Renovato RD, Alvarenga MRM. Representations and uses of medicinal plants in elderly men. Rev Latino-Am Enfermagem. 2012;20(4):778-86.

25.Feijó AM, Bueno MEN, Ceolin T, Linck CL, Schwartz E, Lange $\mathrm{C}$ et al. Plantas medicinais utilizadas por idosos com diagnóstico de Diabetes mellitus no tratamento dos sintomas da doença. Rev bras plantas med. 2012;14(1):50-6.

26. Brasileiro BG, Pizziolo VR, Matos DS, Germano AM, Jamal CM. Plantas medicinais utilizadas pela população atendida no "Programa de Saúde da
Família", Governador Valadares, MG, Brasil. Braz J Pharm Sci. 2008;44(4):629-36.

27. Medeiros MFT, Fonseca VS, Andreata RHP. Plantas medicinais e seus usos pelos sitiantes da Reserva Rio das Pedras, Mangaratiba, RJ, Brasil. Acta Bot Bras. 2004;18(2):391-99.

28.Lorenzetti ER, Conceição DM, Rigotti M, Vieiralves G, Soriano F, Câmara FLA. Estímulo ao Cultivo de Plantas Medicinais e Aromáticas em Botucatu - SP. São Paulo; 2011.

\section{CONFLITO DE INTERESSES}

Os autores declaram não haver conflitos de interesse.

\section{AUTOR PARA CORRESPONDENCIA}

\section{Anderson Martelli}

martellibio@hotmail.com

Submetido em 19/07/2018 Aceito em 04/10/2018 